
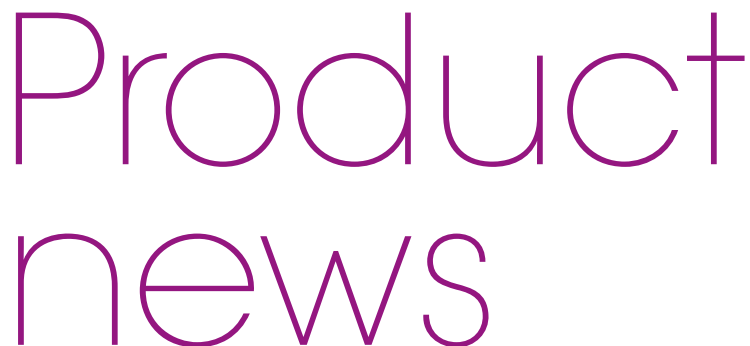

Product news is provided as a service to readers using text and images from the

manufacturer, supplier or distributor and does not imply endorsement by BDJ Team

Normal and prudent research should be exercised before purchase or use of any

product mentioned.

\section{ORAL HYGIENE - MORE THAN JUST BRUSHING}
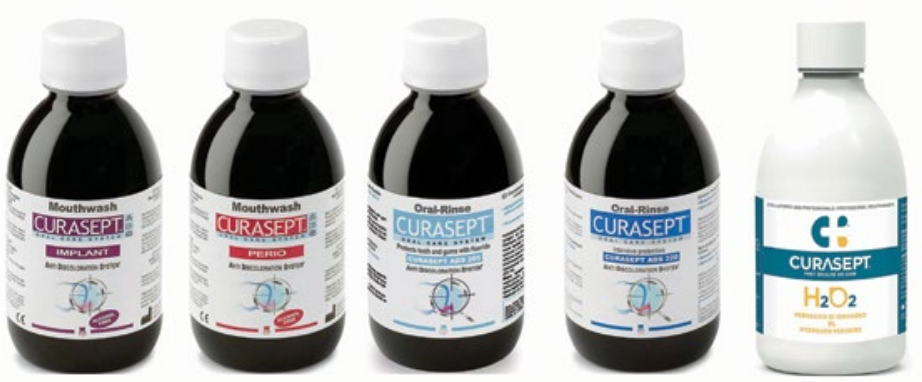

and taste disturbance associated with other solutions. There are also targeted therapies like Curasept ADS Implant and Curasept ADS Perio, which include hyaluronic acid to accelerate healing and PVP-VA to combat future

hor patients to maintain optimal oral brushing their teeth. Why not introduce them to the Curasept ADS range of mouth rinses to take their dental health to the next level?

Available from J\&S Davis, Curasept ADS products contain chlorhexidine for plaque fighting benefits, while being formulated to minimise the risk of staining plaque accumulation.

To help your patients elevate their homecare dental routines, remind them it takes more than just brushing. Recommend Curasept from J\&S Davis.

For more information on the industryleading products available from J\&S Davis, visit www.js-davis.co.uk, call 01438747344 or email jsdsales@js-davis.co.uk.

\title{
NEW RECYCLING PROGRAMME LAUNCHED FOR PRACTICES
}

Every year millions of dental care products end up in landfill and incineration across the UK. To help reduce this, Royal Philips and Terracycle have teamed up to pilot a Dental Care Recycling Programme. The national scheme aims to invite dental professionals to sign up, becoming a place that patients can dispose of their dental waste in an environmentally friendly way. Collecting and recycling these items through The Philips Dental Care Recycling Programme diverts waste from landfill and incineration whilst enabling dental practices to raise money for charitable organisations.

This scheme allows dental professionals the ability to help patients to dispose of waste in an environmentally friendly way, when registered patients next visit their practice. Items that can be recycled include: electric toothbrush heads and covers, electric flosser nozzles, flossing sticks, interdental brushes, and dental floss containers, from all brands.

Dental practices can participate by setting up a free TerraCycle account and submitting a request to receive a recycling collection box. Patients can then drop off their used dental care items in the collection box when they next attend an appointment. When the collection box is full, the dental practice can log into their TerraCycle account and request a prepaid shipping label to send back the collected used dental care products to be recycled. The programme offers 100 TerraCycle points for each kilogram of dental care products sent. However, the points will only be awarded if the
REINFORCE GOOD ORAL CARE HABITS

In the spirit of promoting healthier lifestyle choices this 'Dry January', take the opportunity to reinforce good oral care habits among your patients. You can start by recommending reliable products that can make oral hygiene easier for maximum patient compliance.

Curaprox offers the innovative Hydrosonic Easy electric toothbrush, which comes with a USB charger, a travel case and two brush heads - the CHS 200 sensitive and the CHS 300 power featuring ultra-fine Curen filaments.

Controlled via a single button, the Hydrosonic Easy delivers a powerful hydrodynamic effect with up to 42,000 brush strokes per minute. This facilitates a gentle yet effective clean, even in the most hard-to-reach areas of the mouth.

For more information call 01480 862084, email info@curaprox.co.uk or visit www.curaprox.co.uk.

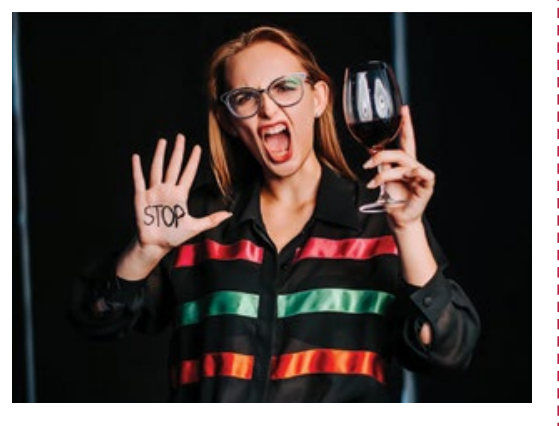

parcel reaches the minimum weight of 2 kilograms.

The points can be redeemed as financial donations to a charity or school of choice.

All waste collected as part of this programme will be shipped to the TerraCycle warehouse where it will be recycled into new products.

This programme already has nearly 200 locations signed up to the scheme and Philips welcomes further dental practices to participate.

To find out more about the programme and to sign up, visit https://www.terracycle. com/en-GB/brigades/philips-uk or https:// www.philips.co.uk/a-w/about/dentalrecycling-scheme.html. 